\title{
The CARIBU gas catcher
}

\author{
G. Savard ${ }^{\mathrm{a}, \mathrm{b}}$, A.F.Levand ${ }^{\mathrm{a}}$, B.J. Zabransky ${ }^{\mathrm{a}}$ \\ ${ }^{a}$ Argonne National Laboratory, Argonne, Illinois, USA \\ ${ }^{b}$ University of Chicago, Chicago, Illinois, USA
}

\begin{abstract}
The CARIBU upgrade of the ATLAS facility provides radioactive beams of neutron-rich isotopes for experiments at low and Coulomb barrier energies. It creates these beam using a large RF gas catcher that collects and cools fission fragments from an intense ${ }^{252} \mathrm{Cf}$ fission source and transforms them into a low-emittance monoenergetic beam. This beam can then be purified, reaccelerated and delivered to experiments. This technique is fast and universal, providing access to all fission fragment species independently of their chemical properties. The CARIBU gas catcher has been built to operate at high ionization density and in the presence of the contamination from the source. A brief overview of the CARIBU concept is given below, together with a more detailed description of the CARIBU gas catcher and the performance it has now achieved.
\end{abstract}

Keywords: radioactive beam, gas catcher, Californium fission

\section{The CARIBU facility}

The CAlifornium Rare Isotope Breeder Upgrade (CARIBU) to ATLAS [1] was conceived to deliver to the low-energy nuclear physics community beams of neutron-rich fission fragments at low and Coulomb barrier energies. These fission fragments play a critical role in the nucleosynthesis of most heavy nuclei [2] and hold the key to the evolution of nuclear structure towards the neutron dripline [3]. Yet, the high-quality mass separated beams of these isotopes that would be necessary to address these issues have not been available for experiments at these energy regimes. CARIBU was built to eliminate the main limitations present in previous facilities by using a fast and universal extraction mechanism, as well as a universal purification technique, to provide access to all species produced in fission. Most of the initial studies on these isotopes, be it masses, lifetimes, decay patterns or basic structure, will not require very high intensity beam but rather a clean beam of modest intensity at the right energy. CARIBU's goal is to provide this for the widest variety of species in this region. The basic concept centers on a low-cost source of fission fragments, in this case spontaneous fission, an RF gas catcher [4] to provide the rapid isotope extraction, and a high-resolution magnetic separator [5] to select the species of interest. A schematized layout of the beam production area is shown in figure 1 .

The fission fragments are obtained from the fission of ${ }^{252} \mathrm{Cf}$. A nominally $1 \mathrm{Ci}$ thin ${ }^{252} \mathrm{Cf}$ source is mounted on one end a few hundred liters large RF gas catcher [6] filled with typically 100 mbar of high purity helium. The fission fragments come out of the fission source as highly-charged ions and lose energy and recollect electrons in the helium gas as they slow down. Once the energy is low enough, they can no longer recapture charges from the helium since helium has a higher binding energy for electrons and as a result they come to rest mainly in charge state +1 or +2 depending on the second ionization potential of the species. This process is very fast, typically microseconds. Once thermalized, the ions have low enough energy to be affected by fields applied inside the gas volume. A combination of DC and RF electric fields guide the ions towards an extraction nozzle located on the gas catcher end opposite to the source, while keeping them from touching any solid surface. The ions reach the nozzle region within a 
few tens of milliseconds and once they are within mm of the nozzle they are overtaken by the gas flow which pulls them outside of the stopping volume to a low pressure area. The ions are then guided through sections of differential pumping by a radio-frequency quadrupole structure that opens into an acceleration region where the pressure is low enough to essentially eliminate the straggling in the acceleration process. There, a 50 $\mathrm{kV}$ electrostatic acceleration stage forms a cooled lowenergy beam from the extracted ions. The whole process, from fission to beam extraction, takes of the order of 20-30 ms.

The ions of interest are then selected in a highresolution isobar separator [5] composed of two 60 degree magnets, electrostatic entrance and exit matching sections, and a higher-order correction electrostatic multipole. The high resolution separator reaches a mass resolution of 1 part in 20000 and yields beams which are dominated by the isotope of interest, although not typically pure. All ions species produced in ${ }^{252} \mathrm{Cf}$ fission are available at this point, with a yield proportional to their fission yield [7] except for the usually negligible decay losses that occur in the 20-30 ms time since fission. The gas catcher and isobar separator systems are located on a large $200 \mathrm{kV}$ high-voltage platform located inside the CARIBU building. The isobar separator is followed by an electrostatic switchyard to determine where the beam will go off the platform. The beam can be transported to a low-energy experimental area, located in the same building, for decay studies or mass measurements, or to ATLAS for post-acceleration via an ECR charge breeder [8] which increases the charge state of the ions for efficient acceleration to 5-10 MeV/u in the ATLAS linac. These beams have similar properties to the stable beams accelerated at ATLAS and can be sent to all experimental stations in the facility. They are used for experiments such as Coulomb excitation with GAMMASPHERE [9] or GRETINA [10] in conjunction with CHICO-II [11], or single-particle structure studies with HELIOS [12].

\section{The CARIBU gas catcher}

CARIBU was an addition to the existing ATLAS superconducting linac for heavy ions. The spontaneous fission of ${ }^{252} \mathrm{Cf}$ was selected as the source of neutronrich isotopes because it provides a different isotope distribution than the standard ${ }^{238} \mathrm{U}$ proton-induced fission used by other facilities and can be operated inexpensively without the use of a second accelerator to induce fission. ${ }^{252} \mathrm{Cf}$ has a halflife of 2.64 years and a fission branch of $3.1 \%$ with the remaining $97 \%$ of the decays going via alpha emission. The few years halflife is short

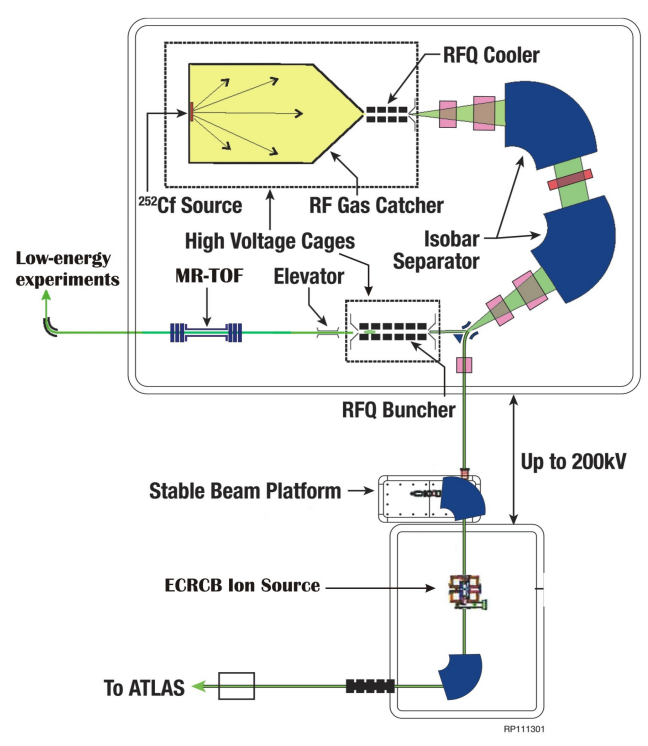

Figure 1: Overview of the CARIBU front end. The top right shows the large high-voltage platform hosting the production area which consist of the source and gas catcher assembly, the isobar separator and the switchyard. On the left of the switchyard is a beamline to a small experimental area for low-energy experiments. The bottom shows the high-voltage platform hosting the ECR charge breeder used to increase the charge state of the radioactive ions before acceleration through ATLAS.

enough to be able to obtain an intense source that is thin enough to avoid self absorbtion, while being long enough to be manageable to work with. The material is available from the HFIR reactor [13] in Oak Ridge. The strongest source that can be handled at an accelerator like ATLAS without reclassification of the facility is 3 $\mathrm{Ci}$, minus the equivalent inventory of other sources already present in the facility. For ATLAS which already hosts other neutron sources for calibration, the limit is effectively 1 to $2 \mathrm{Ci}$ and CARIBU was designed for a $1 \mathrm{Ci}{ }^{252} \mathrm{Cf}$ source, knowing that the source production process can result in significant source strength variation. The gas catcher design for CARIBU therefore needed to be able to handle the intense radiation and ionization produced by this source.

A $1 \mathrm{Ci}^{252} \mathrm{Cf}$ source produces roughly $1 \times 10^{9}$ fission per second, with each fission producing two fission fragments, and $4 \times 10^{10}$ alpha particles per second. The radiation field is approximatively $500 \mathrm{mSv}$ at 30 $\mathrm{cm}$ from the source which means the source, and the gas catcher surrounding it, have to be under very significant neutron and gamma shielding. These are very harsh conditions for operation for a gas catcher. The largest single isotope fission branches are at the $3 \%$ of 


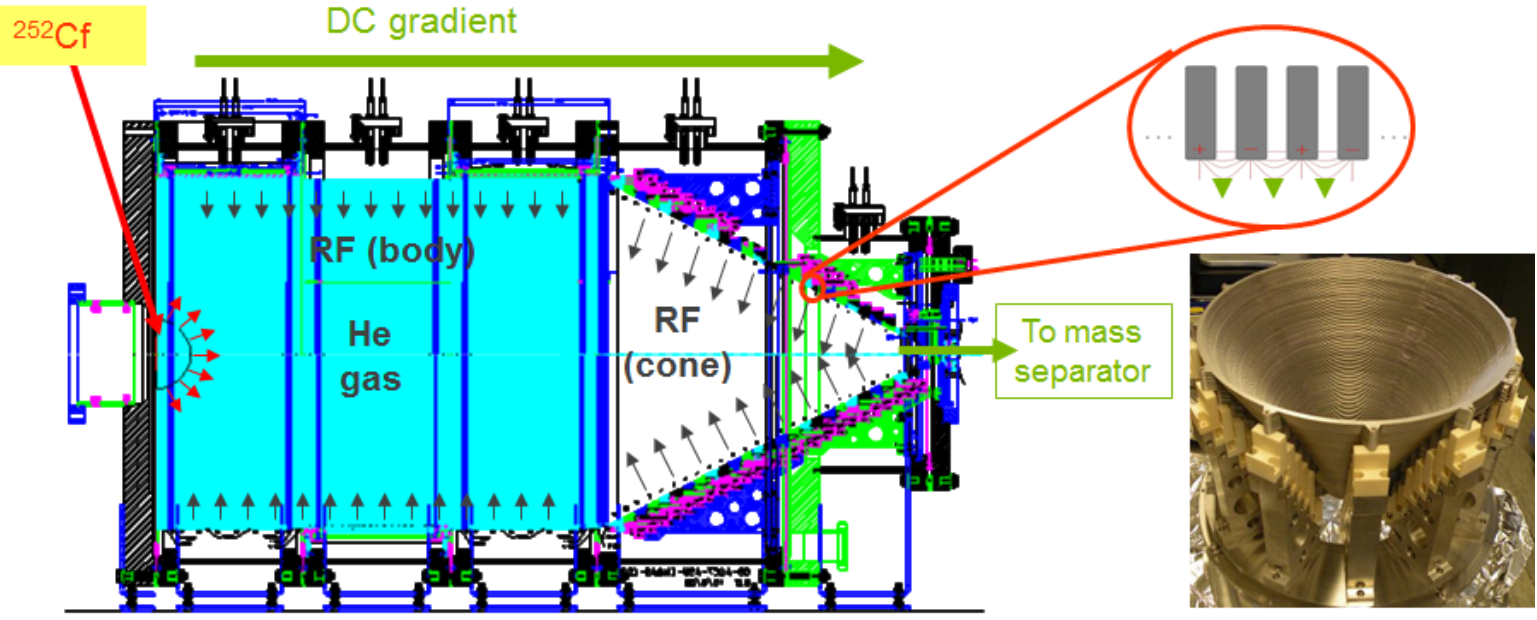

Figure 2: On the left is a drawing of the CARIBU gas catcher showing the body (left) and cone (right) sections and indicating the main forces acting on the ions in the device. At the right is a picture of part (about 250 electrodes) of the RF structure of the cone.

fission level, i.e. roughly one ion of interest per 1000 decays of ${ }^{252} \mathrm{Cf}$. Most reaccelerated beam experiments at Coulomb barrier energy require an intensity of $10^{3}$ to $10^{5}$ ions per second to obtain the minimum information they seek. Since the highest post-acceleration efficiency, including charge breeding, that can be achieved is of the order of $10 \%$, this implies that we require at least $10^{4}$ to $10^{6}$ mass separated ions per second at low energy to reach the required intensities for a successful reaccelerated beam program. The $1 \mathrm{Ci}$ source produces $3-4 \times 10^{7}$ isotopes of interest for the strongest fission branch, with half of them emitted in the $2 \pi$ solid angle facing away from the source so that they can come out of the source. The gas catcher and isobar separator must therefore have a combined efficiency of roughly $10 \%$ or more, under very adverse conditions, to reach the required low-energy (and reaccelerated) yield.

The selected CARIBU gas catcher design uses the linear RF gas catcher [4] approach developed at ANL initially for injection into the CPT [14] mass spectrometer. This approach has been demonstrated to maintain high efficiency at high ionization density in the ANL developments for RIA. The geometry must be adapted for fission fragments which do not have a preferred direction as beam produced isotopes usually have but instead fill the whole $2 \pi$ solid angle out of the source. The modified design is shown in figure 2. The gas catcher is essentially a large cylindrical stopping volume with one end tapered has a cone. It has an inner diameter of $50 \mathrm{~cm}$ and a length of roughly 1.2 meters. The inner surfaces of the volume are made of RF structures (see insert in figure 2) composed of thin successive rings at alternating RF potential. This creates a strong RF field close to the surfaces that repels charged particles. This stops the thermalized ions from neutralizing on the walls of the stopping volume. A DC gradient is also imposed on the RF structure to push the thermalized ions towards the exit nozzle. The chamber is filled with roughly 100 mbar of ultra-high purity ( $i 1$ ppb impurities) helium gas continuously flowing through the gas catcher and evacuated through a nozzle at the end of the cone. The upstream end of the cylinder has an opening to insert the $\mathrm{Cf}$ source, previously mounted on a support plug using remotely controlled manipulators in hot cell facilities, into the gas catcher. The source is covered by a thin $1 \mathrm{mg} / \mathrm{cm}^{2}$ gold foil to minimize the risk of contamination of the gas catcher. The source assembly also sits into a degrader system used to remove some of the energy of the recoils to reduce ionization in the gas. This degrader system also provides a second barrier to minimize contamination from the source inside the gas catcher. Both the source assembly and the degrader system can be removed remotely from the gas catcher so that some contamination of these systems is acceptable (and expected). The gas catcher system itself, with its complex RF structures, cannot be handled remotely and any maintenance needs to be performed manually. The design of the whole system therefore made a priority of limiting possible contamination and eliminating the need for maintenance. The entire gas catcher is made of metal (stainless steel and copper) and ceramics. It contains no components that degrade rapidly in a radiation environment and in principle no maintenance is required in the gas catcher itself.

The gas catcher RF system is split into two main sections: the gas catcher body and the gas catcher cone (see 
figure 2). The gas catcher body section surrounds the main stopping volume. It must transport the thermalized ions towards the cone section while stopping them from diffusing to the walls. This is done by the application of a DC gradient of $10-20 \mathrm{~V} / \mathrm{cm}$ pushing the ions in towards the cone, while applying an RF potential of roughly $150 \mathrm{Vpp}$ to the RF structure to repel ions from the surfaces. In this region, the RF has to stop radial expansion of the ions during transport due to diffusion in the gas and the space charge present in the stopping volume. The DC gradient is perpendicular to the RF structure and does not push the ions towards the surfaces in this region. The pitch (distance between electrode centers) of the RF structure in this region was selected as $1.6 \mathrm{~mm}$. This provides the required RF repulsion while minimizing the capacitance of the structure which therefore also reduces the RF power required to drive it. The gas catcher cone takes the ions delivered by the body section and focuses them to within a few $\mathrm{mm}$ of the nozzle where gas transport takes over. This section must not only repel ions against diffusion and space charge, it must also act against the DC gradient which pushes the ions at roughly 45 degrees towards the surface. A higher $\mathrm{RF}$ repulsion is therefore required. Since the RF voltage amplitude on small structures in helium gas is typically limited to around $150 \mathrm{Vpp}$ (the minimum of the Paschen curve), the higher repulsive force is obtained by reducing the pitch of the RF structure by roughly a factor of 2 in the cone region. The total RF system has over 1000 electrodes and both components are driven by independent resonant circuits with frequencies of $1.5 \mathrm{MHz}$ and $3.4 \mathrm{MHz}$ for the body and cone sections respectively. The total RF power deposited is of the order of 250 Watts.

The 6 tons of radiation shielding required around the gas catcher make the removal of this heat difficult and a complex cooling system that penetrates through the shielding is required. To avoid condensation inside the shielding, the gas catcher is placed inside a vacuum box evacuated to roughly $10^{-3}$ mbar under the shielding. The cooling lines are insulated and follow convoluted paths in the shielding to not affect the neutron shielding. Cooling is provided by a closed-circuit system filled with silicon-based oil that gives an operating range of $-40 \mathrm{C}$ to $+80 \mathrm{C}$. It is used both for cooling the device during operation and heating it for outgassing after openings. The cooling system temperature stabilizes the enclosure of the gas catcher and heat removal from the RF structure is through conduction in the helium gas to the enclosure which is locked at typically $-30 \mathrm{C}$. The resulting temperature on the RF structure is roughly 5 $\mathrm{C}$ higher than the enclosure and after proper outgassing

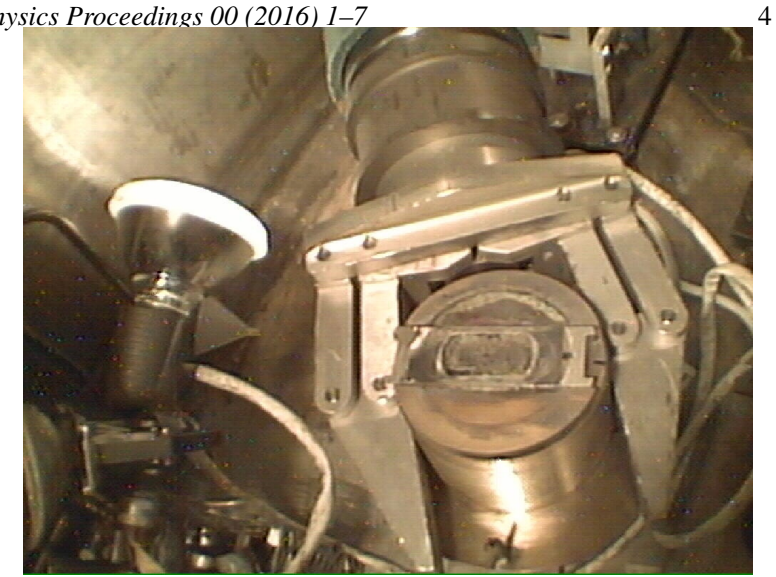

Figure 3: View of the ${ }^{252} \mathrm{Cf}$ deposit held by a manipulator in the hot cell facilities at the HFIR reactor. The source is visible as a roughly 5 $\mathrm{cm}$ by $2 \mathrm{~cm}$ deposit in the center of the support plate. All operations on the source must be done remotely and as a result no cleaning of the plate and deposit for UHV operation is possible.

we see no improvement in yield below -10 C.

The system is built, and all components handled, to UHV standards to not affect the purity of the gas. One component cannot be cleaned to any kind of vacuum standards and that is the ${ }^{252} \mathrm{Cf}$ source itself (see figure 3 ). The source is too active to be handled manually and all operations, from the initial electrodeposition, packaging, shipping, unpacking, installation on the plug and installation in the gas catcher, are done remotely with most of the operations performed in hot cells with manipulators. It is therefore impossible to clean the source. While the deposit takes place on a stainless steel plate, the electrodeposition itself is made from an ammonium acetate solution with a significant amount of material from the solution deposited with the Cf. The vacuum properties of the deposit cannot be easily determined but outgassing from the source deposit was observe to slowly contaminate the gas and decrease performance over periods of weeks. This was resolved by modifying the gas flow patterns inside the gas catcher with the addition of a small pumping channel besides the source plug. The gas flow is only useful for radioactive ion transport at the nozzle where the velocity of the flow increases roughly as $1 / \mathrm{r}^{2}$ and within a few $\mathrm{mm}$ of the nozzle it dominates the DC electric field in directing the ions. In the bulk of the gas catcher volume, the flow speed is low enough that it is nothing more than a small perturbation compared to the DC electric field and changes in the flow patterns there will not affect extraction. The additional small pumping port modifies the flow pattern around the source such that the gas in this region is pumped through the back of the gas catcher, never really mixing with the gas in the bulk of the vol- 
ume, and hence removing with it the contamination that is outgassing from the source. With roughly $10 \%$ of the total flow coming out through the additional aperture, no performance deterioration from the gas catcher is observed with time due to source contamination.

The CARIBU gas catcher main gas flow evacuates through two consecutive sections of radio-frequency quadrupole (RFQ) ion guides, separated by a microRFQ, which then lead to a $50 \mathrm{kV}$ electrostatic acceleration section to form the extracted beam that is fed to the isobar separator. The RFQs have an inner radius of $\mathrm{r}_{0}=7 \mathrm{~mm}$ and can be operated in the frequency range $0.5-2.0 \mathrm{MHz}$. They provide differential pumping by channeling the ions to the extraction region while the helium gas from the gas catcher is pumped by two parallel TS-440 high-flow pumps from Osaka vacuum in the first RFQ section and a $400 \mathrm{l} / \mathrm{s}$ magnetic levitation turbo pump in the second RFQ section. The main backing pump is an SP630 dry screwpump from Leybold. The system typically operates with an 80 mbar pressure of high-purity helium in the gas catcher and a flow of 2 SLM which results in 0.5 mbar in the first RFQ section, $10^{-3}$ mbar in the second RFQ section, and $10^{-6}$ mbar in the acceleration section.

\section{Observed performance}

CARIBU has been in operation for a few years now and over 150 different isotopes have already been extracted for experiments with low-energy and reaccelerated beams. This first radioactive beam facility based on a gas catcher system has demonstrated the expected rapid and universal ion extraction capabilities. Neutronrich isotopes of the elements $\mathrm{Nd}, \mathrm{Ce}, \mathrm{Pr}, \mathrm{La} \mathrm{Ba}, \mathrm{Pm}$, $\mathrm{Sm}, \mathrm{Eu}, \mathrm{Gd}, \mathrm{Rh}, \mathrm{Ru}, \mathrm{Tc}, \mathrm{Mo}, \mathrm{Nb}, \mathrm{Sr}, \mathrm{Cs}, \mathrm{Xe}, \mathrm{I}, \mathrm{Te}$, $\mathrm{Sb}, \mathrm{Sn}$, and In have been extracted with a relative yield essentially in agreement with the tabulated fission yield for ${ }^{252} \mathrm{Cf}$. Most species are extracted preferentially in one charge state, either the $1+$ or $2+$ charge state, depending on the energy of their atomic second ionization potential. A few species, mostly around the Mo region, are extracted with a significant fraction in both charge states. For some species, we also observe a small fraction, typically less than $1 \%$ of the ionic yield, extracted as molecular ions. The extraction time of the system is essentially independent of the ion species, i.e. it appears the same for an easy ISOL beam like cesium and a highly refractory element such as molybdenum, and is calculated to be in the 20-30 ms range under typical operating conditions. While this number cannot easily be measured directly, the observed yield for each isotope follows the expected fission yield, even for isotopes

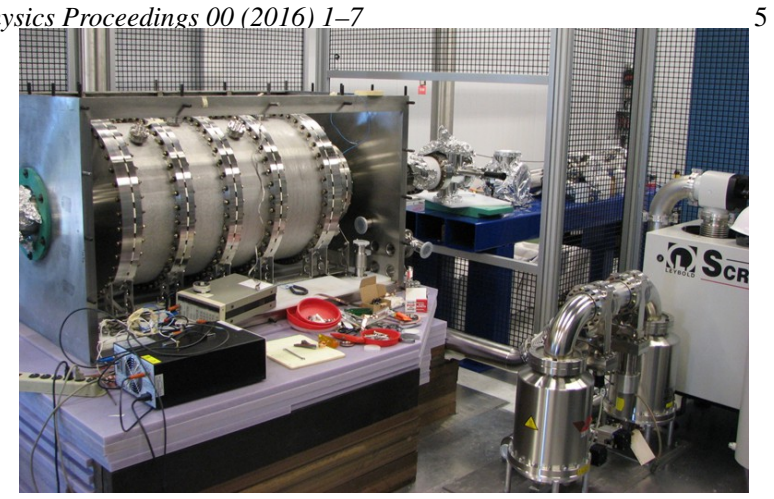

Figure 4: View of the CARIBU gas catcher inside its vacuum enclosure and sitting on part of the borated polyethylene used for neutron shielding. Also visible on the right are the two TS-440 pumps backed by the SP-630 Screwline pump that evacuate the first section of the RFQ cooler.

with lifetime below $100 \mathrm{~ms}$. This is consistent with an extraction time well below $100 \mathrm{~ms}$.

The gas catcher at CARIBU cools and extracts all radioactive species created by californium fission together as one beam which is then mass separated in the isobar separator. It also extracts stable molecular ions created by the ionization deposited by the fission recoils and alpha particles in the gas catcher. The RFQs used to transport the ions through the differential pumping sections provide a weak mass selection that removes the lightest species. The total current extracted from the RFQs is of the order of one to a few nA spread out over more than 100 masses. The total radioactive ion yield cannot be easily measured by ion counting since before mass separation the rate is too high and is dominated by stable ions. After mass separation, the yield of individual species can be determined by measuring the beta-decay rate of collected mass separated samples. This is done at CARIBU with beam intercepting devices composed of a silicon detector covered with a thin aluminum collection foil. These devices provide reliable and normalizable absolute countrates to determine yields and transmission. Such a detector located after the focal point of the isobar separator is used to determine individual mass separated isotope yields. An isotope with a strong fission branch such as ${ }^{143} \mathrm{Ba}$ has a mass separated yield as high as $4 \times 10^{5}$ ions per second in one charge state, the $2+$ state in the case of barium ions, for a fission yield of $4.4 \%$. Essentially all species measured so far have a dominating charge state, either the $1+$ or $2+$ state, with a yield in that charge state proportional to that in Barium corected for the fission branch. The one exception is the isotopes around Molybdenum where the charge state distribution is almost equal between the $1+$ and $2+$ states and where the sum of both charge states scales 
with the barium yield corrected for the fission branch. This allows us to determine the total number of mass separated radioactive ions extracted from the system to be around $1.8 \times 10^{7}$ ions per second. The isobar separator transport efficiency has been determined with stable beam to be of the order of $85 \%$ which means that the number of radioactive ions extracted from the RFQs is $2.1 \times 10^{7}$ per second. The total number extracted from the gas catcher itself will be slightly larger since the RFQs are not $100 \%$ efficient but there is no reliable approach to determine independently their efficiency at CARIBU under battle condition and therefore only a lower limit can be obtained for the number of radioactive ions extracted from the gas catcher itself.

The efficiency of the system at extracting radioactive ions appears essentially independent of ion species since the observed yield follows closely the individual fission branches. Determining this overall efficiency requires knowing how many radioactive ions enter the gas catcher volume per second. This in turn requires knowledge of the source deposit thickness and of the cover foil and degrader the ions must go through before entering the gas volume to determine how many recoils make it through this material. The current ${ }^{252} \mathrm{Cf}$ source was produced at the HFIR reactor in the fall of 2013 and had an estimated activity of $1.76 \mathrm{Ci}$ in December 2013. This source was prepared by electrodeposition on a plate with an approximate $10 \mathrm{~cm}^{2}$ area. This area is larger than the $4 \mathrm{~cm}^{2}$ originally specified for CARIBU but was changed in an attempt to alleviate source thickness issues observed already in the production and use of the weaker sources employed for initial commissioning. Additional material collected together with the ${ }^{252} \mathrm{Cf}$ during electrodeposition in the ammonium acetate solution does not affect the neutron yield but can significantly reduce the amount of fission fragments making it out of the source which is the critical quantity for CARIBU. A simple measurement to estimate source thickness was performed at the HFIR facility before the source was sent to ANL. A sheet thick enough to stop all fission products was positioned a few $\mathrm{mm}$ above the source for a given amount of time and the long-lived activity collected on this foil was subsequently counted after a suitable cooling time. The activity observed for the heavy fission fragments was roughly $11 \%$ of what was expected for a thin source of $1.76 \mathrm{Ci}$. This is a clear indication that the source is much thicker than originally expected. The actual source thickness can be estimated by performing a simulation with the geometry used in the ORNL test and by then varying the effective source thickness to take into account the energy loss in the source until the fission yields match those reported in the test performed at HFIR. The simulations, assuming a homogeneous distribution of the ${ }^{252} \mathrm{Cf}$ activity in the source material, agree with the data for a roughly $38 \mathrm{mg} / \mathrm{cm}^{2}$ total source thickness. The thickness of source material is determined with better than $5 \%$ accuracy in the simulations, but does depend on the choice of source material. However, it was found that this dependence essentially cancels out when performing the simulations of stopping in the gas catcher (described below) postulating the same source material. This thickness implies that the source contains roughly two orders of magnitude more material than the ${ }^{252} \mathrm{Cf}$ itself as the $1.7 \mathrm{Ci}$ activity should correspond to a thickness of $0.32 \mathrm{mg} / \mathrm{cm}^{2}$ for a deposition over a $10 \mathrm{~cm}^{2}$ area. The source is expected to contain known amounts of other $\mathrm{Cf}$ and $\mathrm{Cm}$ isotopes so that the total actinide areal mass density should have been roughly twice the ${ }^{252} \mathrm{Cf}$ amount. This leaves a large amount of material contributing to the target thickness unaccounted for. The exact nature of this additional material present in the source has not been clearly identified (this is difficult for a sample with such a large activity), but the amount is so large that it probably cannot come from anything but the ammonium acetate solution itself.

The effect of the additional, unwanted source thickness on CARIBU yields can be evaluated by performing a simulation of ${ }^{144} \mathrm{Ba}$ ions stopping in the CARIBU gas catcher using the determined source thickness as input. In CARIBU, the source is installed on the shielding plug and is covered by a $1 \mathrm{mg} / \mathrm{cm}^{2} \mathrm{Au}$ foil and a shaped degrader made of thin Al foils. The Au cover foil is present to prevent source sputtering from contaminating the main gas catcher volume and provides containment of any flaking from the source to the region just next to it. The plug holding the source and the cover foil are the only components of CARIBU that are fully handled remotely and, hence, are designed to tolerate significant contamination. The integrity of the cover foil is critical to minimize spreading of any contamination to other CARIBU components, and $\mathrm{Au}$ foils with $1 \mathrm{mg} / \mathrm{cm}^{2}$ thickness have been found to best survive in this environment. Such a thickness is required to accommodate the mechanical forces exerted when the source is moved in and out of the gas catcher. The degrader is mounted on a second holder connected to the plug, and is used to optimize the stopping of recoiling fragments in the gas catcher while at the same time minimizing the total ionization created by these recoils in the helium volume. It also constitutes a secondary confinement region in the event that the Au cover foil is damaged. The degrader shape resembles that of a top hat of $10 \mathrm{~cm}$ diameter and roughly $5 \mathrm{~cm}$ height cen- 
tered on the source, with a thickness that was decreased to just under $2 \mathrm{mg} / \mathrm{cm}^{2} \mathrm{Al}$ once it was determined that the source was thicker than expected. This geometry was entered into the simulation in order to determine the fraction of the recoiling ions emitted into a $2 \pi$ solid angle from the source that could make it out of this assembly and enter into the gas catcher stopping volume. At a thickness of $38 \mathrm{mg} / \mathrm{cm}^{2}$; i.e., the thickness required to reproduce the measurement made at HFIR , the loss (with respect to original estimates) in the number of ions making it through the cover foil and degrader assembly entering the gas catcher is roughly a factor of 19 . With this information, one can then calculate the expected number of ions from the present source. The source had an activity of $1.76 \mathrm{Ci}$ in December 2013, which translates into $1.21 \mathrm{Ci}$ at the time of the yield measurements presented above. The effective source strength at that time, correcting for the source thickness, was therefore $1.21 / 19=0.064 \mathrm{Ci}$. Taking this number, together with the fact that only the ions emitted in the $2 \pi$ solid angle out of the source can enter the gas catcher, yield 8.5 $\mathrm{X} 10^{7}$ radioactive ions entering the gas catcher volume per second and an average extraction efficiency for the combined gas catcher and RFQ coolers of $25 \%$.

The degrader system was also meant originally to be tuned to the range of specific isotopes after the fission process. With a thin source, this allows to optimize the fraction stopping in the gas for a given amount of energy deposition. With the large thickness of the currently available source, little energy and hence range difference between the various species remains and this feature cannot be used at this point. Otherwise, the CARIBU gas catcher is working essentially as expected.

\section{Status}

CARIBU is now operational and provides a broad array of neutron-rich isotopes at low and Coulomb barrier energies for experiments in nuclear structure, astrophysics and applications. The concept, centered on the fast, efficient and universal extraction provided by a large gas catcher, works essentially as expected. The CARIBU gas catcher is operating with high-efficiency with $10^{8}$ radioactive ions per second and close to $10^{10}$ alpha particles per second entering the stopping volume per second. The main limitation at this point comes from the Californium source itself which is much thicker than expected, resulting in only a small fraction of the recoils actually escaping from the deposit. R\&D is progressing at HFIR to obtain a thinner deposit and a new source, hopefully a thinner one, will be available sometimes early next year.

\section{Acknowledgment}

This work was supported by the U.S. Department of Energy, Office of Nuclear Physics, under Contract No. DE-AC02-06CH11357.

\section{References}

\section{References}

[1] G. Savard, R.C. Pardo, S. Baker, C.N. Davids, et al., Hyperfine Interact. 199 (1-3) (2011) 301-309.

[2] R. Surman et al., Eur. Phys. Jour. Web Conf. 66 (2014) 07024.

[3] J. Erler et al., Nature 486 (2012) 509.

[4] G. Savard et al., Nucl. Instr. and Meth. B204, 582 (2003).

[5] C. Davids and D. Peterson, Nucl. Instr. and Meth. B266, 19 (2008).

[6] G. Savard, J. Phys. : Conf. Ser. 312 (2011) 052004.

[7] T.R. England and B.F. Rider, Los Alamos technical report LAUR-94-3106, http:/ie/lbl.gov/fission/252Cf.txt .

[8] R. Vondrasek et al., Rev. of Sci. Instr. 82 (2011) 053301

[9] I.-Y. Lee, Prog. Part. Nucl. Phys. 28, 473 (1992).

[10] M.A. Deleplanque et al., Nucl. Instr. and Meth. A430, 292 (1999).

[11] M.W. Simon et al., Nucl. Instr. and Meth. A452, 205 (2000).

[12] A.H. Wuosmaa et al., Nucl. Instr. and Meth. A580, 1290 (2007).

[13] https://neutrons.ornl.gov/hfir

[14] G. Savard et al., Hyperfine Interact. 132, 221 (2001). 